Ferrata Storti Foundation

\title{
Azacitidine with or without lenalidomide in higher risk myelodysplastic syndrome \& low blast acute myeloid leukemia
}

Haematologica 2019

Volume 104(4):700-709

\author{
Melita Kenealy, ${ }^{1,2}$ Mark Hertzberg, ${ }^{3}$ Warwick Benson, ${ }^{4}$ Kerry Taylor, ${ }^{5}$ \\ Ilona Cunningham, ${ }^{6,7}$ Will Stevenson, ${ }^{8}$ Devendra Hiwase, ${ }^{9,10,11}$ Richard Eek, ${ }^{12}$ \\ Daniela Zantomio, ${ }^{13}$ Steve Jong, ${ }^{14}$ Meaghan Wall, ${ }^{15,16,17}$ Piers Blombery, ${ }^{18,19}$ \\ Tracey Gerber, ${ }^{20}$ Marlyse Debrincat, ${ }^{20,21,22}$ Diana Zannino ${ }^{20}$ and John F. \\ Seymour ${ }^{18,23}$
}

${ }^{1}$ Cabrini Health, Melbourne; ${ }^{2}$ Monash University, Melbourne; ${ }^{3}$ Prince of Wales Hospital, Randwick, Sydney; ${ }^{4}$ Westmead Hospital, Sydney; ${ }^{5}$ con Cancer Care, Brisbane; ${ }^{6}$ Concord Hospital University of Sydney; ${ }^{7}$ University of Sydney; ${ }^{8}$ Royal North Shore Hospital, St Leonards; ${ }^{9}$ Haematology Department, Royal Adelaide Hospital; ${ }^{10}$ School of Medicine, Univeristy of Adelaide; ${ }^{11}$ Cancer Theme, South Australian Health and Medical Research (SAHMRI), Adelaide; ${ }^{12}$ Border Medical Oncology, Albury; ${ }^{13}$ Austin Health, Melbourne; ${ }^{14}$ Andrew Love Cancer Centre, University Hospital, Geelong; ${ }^{15}$ Victorian Cancer Cytogenetics Service, St Vincent's Hospital, Fitzroy, Victoria; ${ }^{16}$ Department of Medicine, St Vincent's Hospital, University of Melbourne, Fitzroy, Victoria; ${ }^{17}$ St Vincent's Institute of Medical Research, Fitzroy, Victoria; ${ }^{18}$ Peter MacCallum Cancer Centre, Melbourne; ${ }^{19} \mathrm{Sir}$ Peter MacCallum Department of Oncology, University of Melbourne; ${ }^{20}$ Australasian Leukaemia and Lymphoma Group, Richmond; ${ }^{21}$ Systems Biology and Personalised Medicine Division, The Walter and Eliza Hall Institute of Medical Research, Melbourne; ${ }^{22}$ Department of Medical Biology, University of Melbourne and ${ }^{23}$ University of Melbourne, Australia

\section{Correspondence:}

MELITA KENEALY

melita.kenealy@thebloodunit.com.au

Received: July 10, 2018.

Accepted: November 23, 2018.

Pre-published: December 13, 2018.

doi:10.3324/haematol.2018.201152

Check the online version for the most updated information on this article, online supplements, and information on authorship \& disclosures: www.haematologica.org/content/104/4/700

(C)2019 Ferrata Storti Foundation

Material published in Haematologica is covered by copyright. All rights are reserved to the Ferrata Storti Foundation. Use of published material is allowed under the following terms and conditions:

https://creativecommons.org/licenses/by-nc/4.0/legalcode. Copies of published material are allowed for personal or internal use. Sharing published material for non-commercial purposes is subject to the following conditions:

https://creativecommons.org/licenses/by-nc/4.0/legalcode, sect. 3. Reproducing and sharing published material for commercial purposes is not allowed without permission in writing from the publisher.

\section{ABSTRACT}

S tandard treatment for higher risk myelodysplastic syndromes, chronic myelomonocytic leukemia and low blast acute myeloid leukemia is azacitidine. In single arm studies, adding lenalidomide had been suggested to improve outcomes. The ALLG MDS4 phase II trial randomized such patients to standard azacitidine or combination azacitidine $\left(75 \mathrm{mg} / \mathrm{m}^{2} / \mathrm{d}\right.$ days 1 to 5$)$ with lenalidomide (10mg days $1-21$ of 28-day cycle from cycle 3 ) to assess clinical benefit (alive without progressive disease) at 12 months. A total of 160 patients were enrolled; median age 70.7 years (range $42.5-87.2$ ), 31.3\% female with 14\% chronic myelomonocytic leukemia, $12 \%$ acute myeloid leukemia and $74 \%$ myelodysplastic syndromes. Adverse events were similar in both arms. There was excellent delivery of protocol therapy (median azacitidine cycles 11 both arms) with few dose reductions, delays or early cessations. At median follow up 33.1 months (range 0.7-59.5), the rate of clinical benefit at 12 months was $65 \%$ azacitidine arm and $54 \%$ lenalidomide+azacitidine $\operatorname{arm}(P=0.2)$. There was no difference in clinical benefit between each arm according to WHO diagnostic subgroup or IPSS-R. Overall response rate was $57 \%$ in azacitidine arm and $69 \%$ in lenalidomide+azacitidine $(P=0.14)$. There was no difference in progression- free or overall survival between the arms (each $P>0.12$ ). Although the combination of lenalidomide and azacitidine was tolerable, there was no improvement in clinical benefit, response rates or overall survival in higher risk myelodysplastic syndrome, chronic myelomonocytic leukemia or low blast acute myeloid leukemia patients compared to treatment with azacitidine alone. This trial was registered at www.anzctr.org.au as ACTRN12610000271000. 


\section{Introduction}

The myelodysplastic syndromes (MDS) and chronic myelomonocytic leukemia (CMML), an MDS/myeloproliferative neoplasm overlap syndrome, are a group of clonal bone marrow disorders characterized by active but ineffective and clonal hematopoiesis accompanied by morphological dysplasia and variable cytopenias. Cytogenetic abnormalities and/or recurrent somatic mutations are present in the majority of cases. ${ }^{1,2}$ The prognosis is variable with $30 \%$ of patients transforming to acute myeloid leukemia (AML) ${ }^{2,3}$ AML with a "low blast" count of 20$29 \%$ has a similar prognosis to MDS with blasts of 10$19 \%{ }^{4}$ The most widely used tool for stratifying clinical risk in MDS is the IPSS score. ${ }^{4,5}$

Azacitidine is approved and available for use in subsets of intermediate- to high-risk MDS. It is a nucleoside analogue that has direct cytotoxicity and gives rise to DNA hypomethylation through interference with DNA methyltransferase. ${ }^{6}$ Clinical responses are manifest by an improvement in hematologic parameters and quality of life in a broad population of MDS patients including those with lower-risk disease but significant cytopenias. ${ }^{7,8}$ Overall survival is prolonged in those with higher-risk disease. ${ }^{9}$ There is also an established role for azacitidine in low-blast count AML and elderly AML with $>30 \%$ BM blasts. ${ }^{10,11}$ Azacitidine is an established standard of care in these patients, but even so the disease does not respond in many patients and survival remains suboptimal. Ball et al. reviewed a number of studies that combined hypomethylating agents (azacitidine and decitabine) with a number of different medication classes including small molecules, immunomodulators and monoclonal antibodies, but found a lack of survival advantage in these combinations compared to HMA monotherapy. ${ }^{12}$ Emerging data suggests molecular profiles may influence response to azacitidine. ${ }^{13}$

Lenalidomide is a thalidomide analogue and is both more potent and tolerable relative to thalidomide..$^{14,15}$ Its efficacy in MDS is most pronounced in patients with $5 \mathrm{q}-$ MDS (low risk MDS). ${ }^{16}$ Targeted degradation of CK1a (encoded by the retained allele of CSNK1A1 at 5q32 in cells with $5 \mathrm{q}-$ ) achieves cytogenetic remission and transfusion independence in the majority of patients. ${ }^{15}$ Clinically relevant responses are also seen in lower-risk disease without $5 q_{-}-{ }^{17,18,19}$ In MDS without $5 q^{-}$, the primary mechanism of disease control with lenalidomide appears to be immunomodulation. ${ }^{14}$ Defective or reduced immune interaction between host and tumor contributes to the pathogenesis of MDS. Lenalidomide overcomes this by reducing pro-inflammatory cytokines, upregulation of $\mathrm{T}$ - and NK-cell activity and inhibition of angiogenic activity. These effects prevent apoptosis of healthy stem cells, improve erythropoiesis and direct immune responses against abnormal hematopoietic clones. ${ }^{14}$

The combination of a demethylating agent and an immunomodulatory drug has been explored in phase-I and -II studies in MDS, CMML and low blast AML in an attempt to improve outcomes. The ALLG MDS3 trial of azacitidine and thalidomide ${ }^{20}$ showed promising response rates, and a phase-II study by Sekeres et al. ${ }^{21}$ including the combination of azacitidine and lenalidomide in higherrisk MDS (blasts $\geq 5 \%$ or IPSS $\geq 1.5$ ) or CMML resulted in an overall response rate of $49 \%$ compared to $38 \%$ azacitidine alone $(P=0.14)$, with the subgroup of CMML patients on combination therapy achieving an improved ORR compared to aza alone ( $68 \%$ vs. $28 \%, P=0.02)$. Other groups have gone on to review the safety and efficacy of this combination in similar disease groups; elderly AML patients and high risk MDS and AML with $\leq 30 \%$ blasts. ${ }^{22,23}$ Narayan et al. demonstrated a modest 25\% response rate in elderly patients with previously treated AML and high-risk MDS. In these responders, the overall survival was 9.6 months compared to 4 months for non responders. ${ }^{22}$

We conducted an open-label, multicentre randomized phase-II study across 30 sites in Australia to assess the efficacy of azacitidine in combination with lenalidomide compared to standard azacitidine alone in the treatment of higher-risk MDS, CMML and low blast AML.

\section{Methods}

\section{Study design and treatment}

ALLG MDS4 was an open-label, multi-centre study conducted across 30 Australian sites. The study was registered at anzctr.org.au ACTRN12610000271000, was reviewed and approved by the Human Research Ethics Committees of each centre and conducted according to the Declaration of Helsinki. All patients provided written informed consent prior to participation.

The primary objective was to demonstrate improved efficacy with the combination compared to azacitidine alone. Secondary objectives were to describe response rates, response duration, overall survival, tolerability and changes in quality of life, and to explore biomarkers of response and mechanism of action of azacitidine and lenalidomide.

Patients were stratified according to IPSS (low-Int1 or Int2high), ${ }^{5}$ by centre and by disease category (MDS, AML or CMML), ${ }^{24}$ and randomized 1:1 to either azacitidine alone at standard dosing of $75 \mathrm{mg} / \mathrm{m}^{2} / \mathrm{d} \times 7$ days (on a 5-2-2 interrupted sched$\mathrm{ule}^{25}$ ) each 28 day cycle subcutaneously, or to the combination azacitidine plus lenalidomide. Patients on the combination arm received azacitidine alone at the above dose and schedule for the first 2 cycles, then commenced lenalidomide $10 \mathrm{mg} / \mathrm{d}$ from day 1 of cycle 3 with a reduction in azacitidine dose with the combination to $75 \mathrm{mg} / \mathrm{m}^{2} / \mathrm{d}$ for 5 consecutive days per cycle as per phase 1 data available at the time. ${ }^{26}$ The rationale for this was to limit the expected myelotoxicity typically seen in the first 2 cycles of treatment with azacitidine and so to improve the deliverability of combination treatment. Lenalidomide was continued only until completion of C12 due to limited data on longer-term combination toxicity. Azacitidine as a single agent was continued after the primary endpoint assessment at 12 months, until disease progression or unacceptable toxicity. Patients were followed for transformation to AML and survival until the last registered patient had been followed for a minimum 2 years after completion of the first 12 months of treatment.

\section{Patient population}

Patients were eligible with a diagnosis of non-proliferative CMML, AML with blasts $<30 \%$ or MDS by WHO criteria; ${ }^{24}$ those with refractory cytopenia with unilineage dysplasia (RCUD) and refractory anemia with ringed sideroblasts (RARS) had to have at least one clinically significant cytopenia as defined in the protocol (refer Online Supplementary Appendix), consistent with early studies of azacitidine in a broader group of patients with MDS? Patients were 18 years or older and could have either de novo or secondary disease. They must have received no prior chemotherapy for MDS or AML except low dose cytarabine or hydroxyurea, 
and no prior demethylating agent or immunomodulatory drug. They were to have a performance status of ECOG 0-2 and adequate organ function (Online Supplementary Appendix). GCSF was only used for short term management of severe neutropenic infections with no response assessment performed within 21 days of use. Patients on a stable dose of EPO prior to study entry were allowed to continue unchanged while on study.

\section{Statistical plan}

Analyses were carried out using the SAS (Statistical Analysis System, Version 9.3, SAS Institute, North Carolina, USA) software and graphs were produced in $\mathrm{R}$ version 3.2.3 software ( $\mathrm{R}$ Foundation for Statistical Computing, Vienna, Austria, http://www.R-project.org). All comparisons were by intention to treat. The close-out date for this analysis was $12^{\text {th }}$ March 2016.

A sample size of 160 patients (80 per arm) would provide $90 \%$ power, assuming a two-sided type I error of $5 \%$, to detect an improvement of at least $25 \%$ in clinical benefit at 12 months, where the expected rate of clinical benefit at 12 months in the control arm of $50 \%$, given a median time to progressive disease, relapse or death in the AZA001 study of 14.1 months, ${ }^{9}$ and an expected rate of clinical benefit in the combination arm being $75 \%$.

\section{Toxicity}

Adverse event rates are based on the worst grade reported during study treatment for those patients who commenced treatment. Fisher's exact test was used to compare adverse event rates between the randomized arms. All events and grades are based on the CTCAE v4.0 unless otherwise specified. Emerging grade 3+ haematologic toxicity applied to patients who did not have a haematologic toxicity at baseline but developed whilst on treatment. Results are based on absolute value from the screening averaged haematology counts. A grade 3+ toxicity for neutrophil and platelet data was defined as a reduction of more than $50 \%$ from baseline but for haemoglobin Grade 3+ was defined as $\mathrm{Hb}<80 \mathrm{~g} / \mathrm{L}$.

\section{Efficacy}

All patients who were randomized (intention to treat group) were considered in efficacy analysis. 2006 IWG criteria were used for all responses. ${ }^{27}$ The primary endpoint was "clinical benefit at 12 months", defined as the patient being alive and progression/relapse free at 12 months (+/- 1 month) post commencement of treatment, and so included those patients with stable disease at 12 months as achieving clinical benefit. Best response was determined using all assessments performed at the commencement of each cycle from C3 until treatment discontinuation, with bone marrow biopsies performed after C2, C4, C8 and $\mathrm{C} 12$. The overall response rate (ORR) included all patients achieving improvement $(\mathrm{HI})$, marrow $\mathrm{CR}, \mathrm{PR}$ and $\mathrm{CR}$ as best response.

Univariable logistic regression models were used to assess the impact of the following pre-defined variables on response (marrow CR or better): treatment arm, IPSS-R, IPSS, cytogenetic risk group, WHO diagnosis (MDS vs. AML vs. CMML).

Progression-free survival (PFS) was measured from the commencement of treatment to disease progression or death from any cause. Overall survival (OS) was measured from the commencement of treatment to death from any cause. OS and PFS duration was censored at the study close-out date for patients who were still being followed up without having experienced the relevant event by the close-out date, or at the date of last contact for patients who were lost to follow up before the study close-out date. The Kaplan-Meier (product-limit) method was used to estimate PFS and OS and median follow-up time (using the censoring distribution). The logrank test was used to compare survival between the treatment arms and IPSS-R subgroups.

\section{Quality of life (QoL)}

The EORTC OLO C30 was utilized to describe differences in QoL parameters. These analyses were performed on five functional scales (physical, role, emotional, social and cognitive), three symptom scales (fatigue, nausea \& vomiting and pain) and a global health status/QoL scale and six single items. Regression methods accounting for repeated measures (i.e., generalized estimating equations (GEE) with an exchangeable correlation structure) were used to estimate the difference between the treatment arms adjusted for baseline QoL score and weeks on trial. Differences between arms are expressed such that positive differences favour the LEN+AZA arm and negative differences favour the AZA arm.

\section{Molecular and Biomarkers}

Next generation sequencing (NGS). Sequence analysis of targeted regions within 26 genes involved in myeloid malignancy (Peter MacCallum Cancer Centre Myeloid Amplicon Panel (v5.4)) was performed in duplicate using Access Array methodology (Fluidigm, South San Francisco, CA, USA) to prepare ampliconbased, indexed libraries that were sequenced to a depth of $\sim 1000$ reads per amplicon on a MiSeq instrument using v2 chemistry (Illumina, San Diego, CA, USA). Alignment, variant calling and annotation were performed using a custom pipeline. Variants were evaluated using multiple functional and quality filters to identify likely pathogenic variants.

SNP-Array testing. DNA (200 ng) was hybridized to CytoSNP-12 BeadChip arrays (Illumina, San Diego, CA) according to the manufacturer's instructions. Analysis was performed using Karyostudio v1.4 software (Illumina). Karyotypes were reported according to the International System for Cytogenetic Nomenclature (ISCN 2013).

Exploratory analyses include changes in promoter DNA methylation during cycle 1 and at additional time points on treatment, immunophenotyping of MDS population, T-cell subsets, NK-cell function and cytokine profile as predictors of response to treatment and will be reported separately.

\section{Results}

Baseline demographics and disease features (Table 1)

One hundred and sixty patients with a median age of 70.7 years (range 42.5-87.2) were enrolled on study between August 2010 and August 2012; 159 received study drug. The median time from diagnosis to treatment was 1.0 year (0.0-13.2). Twenty-two patients $(14 \%)$ had CMML, 19 (12\%) AML and the remaining 74\% MDS were mostly RCMD or RAEB $1 / 2$ subtypes. Overall IPSS was Low-Int 1 in $61 \%$; by IPSS-R Very Low/Low/Intermed in $63 \%$ with no difference in prognostic or cytogenetic subgroups between arms. Fiftyseven percent of patients were transfusion dependent at study entry. The $5 q$ - cytogenetic abnormality was present as an isolated abnormality in only 3 patients ( IPSS Int- 1 in 2 patients, Int- 2 in 1).

\section{Molecular characteristics at baseline}

Targeted amplicon sequencing and SNP-A testing was successfully performed in 66 cases. Targeted amplicon sequencing detected pathogenic mutations in one or more genes in $94 \%(62 / 66)$ of patients and SNP-array detected 
abnormalities in $50 \%$ (33/66) of patients consistent with published literature. ${ }^{28,29}$ Of the 4 patients without abnormalities detectable by NGS, 1 patient had monosomy 7 detectable on SNP-A resulting in $95.4 \%$ (63/66) of cases having a detectable molecular aberration. The average number of SNP-A abnormalities per case was 7 (range 037). Identification of additional SNP-A abnormalities upstaged cytogenetic risk in 24\% (16/65) cases (G-banded karyotype not available in one case). SNP-A and mutational data are summarized in Table 2.

Table 1. Clinical and hematologic characteristics of 160 patients by assigned treatment cohort.

\begin{tabular}{|c|c|c|c|}
\hline Baseline characteristics & AZA $(n=80)$ & LEN+AZA $(n=80)$ & Total (n=160) \\
\hline Age years, median (range) & $69.1(42.5-85.9)$ & $71.4(44.1-87.2)$ & $70.7(42.5-87.2)$ \\
\hline Male & $52(65)$ & $58(73)$ & $110(68.8)$ \\
\hline Female & $28(35)$ & $22(28)$ & $50(31.3)$ \\
\hline ECOG 0 & $42(53)$ & $41(51)$ & $83(51.9)$ \\
\hline 1 & $36(45)$ & $33(41)$ & $69(43.1)$ \\
\hline 2 & $2(3)$ & $6(8)$ & $8(5.0)$ \\
\hline \multicolumn{4}{|l|}{ Diagnosis WHO } \\
\hline RCUD (RA, RN, RT) & $1(1)$ & $0(0)$ & $1(0.6)$ \\
\hline RARS & $6(8)$ & $3(4)$ & $9(5.6)$ \\
\hline RCMD & $24(30)$ & $28(35)$ & $52(32.5)$ \\
\hline RAEB-1 & $11(14)$ & $11(14)$ & $22(13.8)$ \\
\hline RAEB-2 & $16(20)$ & $15(19)$ & $31(19.4)$ \\
\hline MDS-U & $0(0)$ & $1(1)$ & $1(0.6)$ \\
\hline MDS isolated del5q- & $2(3)$ & $1(1)$ & $3(1.9)$ \\
\hline CMML & $12(15)$ & $10(13)$ & $22(13.8)$ \\
\hline AML & $8(10)$ & $11(14)$ & $19(11.9)$ \\
\hline \multicolumn{4}{|l|}{ IPSS risk group } \\
\hline Low & $12(15)$ & $10(13)$ & $22(13.8)$ \\
\hline Int-1 & $37(46)$ & $38(48)$ & $75(46.9)$ \\
\hline Int-2 & $22(28)$ & $17(21)$ & $39(24.4)$ \\
\hline High & $9(11)$ & $15(19)$ & $24(15.0)$ \\
\hline \multicolumn{4}{|l|}{ IPSS-R risk group } \\
\hline Very Low & $2(3)$ & $3(4)$ & $5(3.3)$ \\
\hline Low & $24(32)$ & $18(24)$ & $42(28.0)$ \\
\hline Intermediate & $23(31)$ & $25(33)$ & $48(32.0)$ \\
\hline High & $16(21)$ & $12(16)$ & $28(18.7)$ \\
\hline Very High & $10(13)$ & $17(23)$ & $27(18.0$ \\
\hline missing & 5 & 5 & 10 \\
\hline \multicolumn{4}{|l|}{ Cytogenetics (IPSS-R) } \\
\hline Very Good & $1(1)$ & $2(3)$ & $3(2.0)$ \\
\hline Good & $55(73)$ & $51(68)$ & $106(70.7)$ \\
\hline Intermed & $11(15)$ & $11(15)$ & $22(14.7)$ \\
\hline Poor & $3(4)$ & $2(3)$ & $5(3.3)$ \\
\hline Very poor & $5(7)$ & $9(12)$ & $14(9.3)$ \\
\hline Carrying 5q- & $10(13)$ & $13(17)$ & $23(15.3)$ \\
\hline missing & 5 & 5 & 10 \\
\hline \multicolumn{4}{|l|}{ Past therapy } \\
\hline EPO & $3(4)$ & $0(0)$ & $3(1.9)$ \\
\hline GCSF & $2(3)$ & $2(3)$ & $4(2.5)$ \\
\hline Low dose cytarabine & $0(0)$ & $1(1)$ & $1(0.6)$ \\
\hline \multicolumn{4}{|l|}{ Baseline cytopenias } \\
\hline $\mathrm{Hb}(<100 \mathrm{~g} / \mathrm{L})$ & $57(71)$ & $53(66)$ & $110(68.8)$ \\
\hline Neutrophils $\left(<1.5 \times 10^{9} / \mathrm{L}\right)$ & $47(59)$ & $37(46)$ & $84(52.5)$ \\
\hline Platelet $\left(<100 \times 10^{9} / \mathrm{L}\right)$ & $42(53)$ & $48(60)$ & $90(56.3)$ \\
\hline
\end{tabular}

Hb: hemoglobin; ECOG: Eastern Cooperative Oncology Group; Int: intermediate; MDS-(U): myelodysplastic syndrome-(unclassifiable); RA: refractory anemia, RAEB: refractory anemia with excess blasts; RCMD: refractory cytopenia with multilineage dysplasia; RARS: refractory anemia with ringed sideroblasts; RCUD: refractory cytopenia with unilineage dysplasia; RN: refractory neutropenia; RT: refractory thrombocytopenia;WHO: World Health Organisation. Median (range) reported and N (\%) unless otherwise specified. 
Most CMML cases $(5 / 7,71 \%)$ had a normal SNP-A karyotype. Rates of SNP-A karyotypic complexity and $\operatorname{del}(5 q)$ were highest in RAEB-2 $(7 / 16,43 \%)$ and AML (2/4, 50\%). Both AML cases with an abnormal karyotype also had deletion of $17 \mathrm{p}$. There were no significant differences between the AZA and AZA+LEN groups in terms of high-risk molecular profile, SNP-A complexity or cytogenetic risk group. Clinical benefit was most frequent in the normal SNP-A $(29 / 39,82 \%)$ and IPPS-Rsnp good cytogenetic risk group $(28 / 39,82 \%)$ cases.

\section{Treatment}

With a close out date of $12^{\text {th }}$ March 2016, median follow up was 33.1 months (range 0.7-59.5). There was excellent drug delivery, with the median number of azacitidine cycles per patient administered of 11 in both arms with
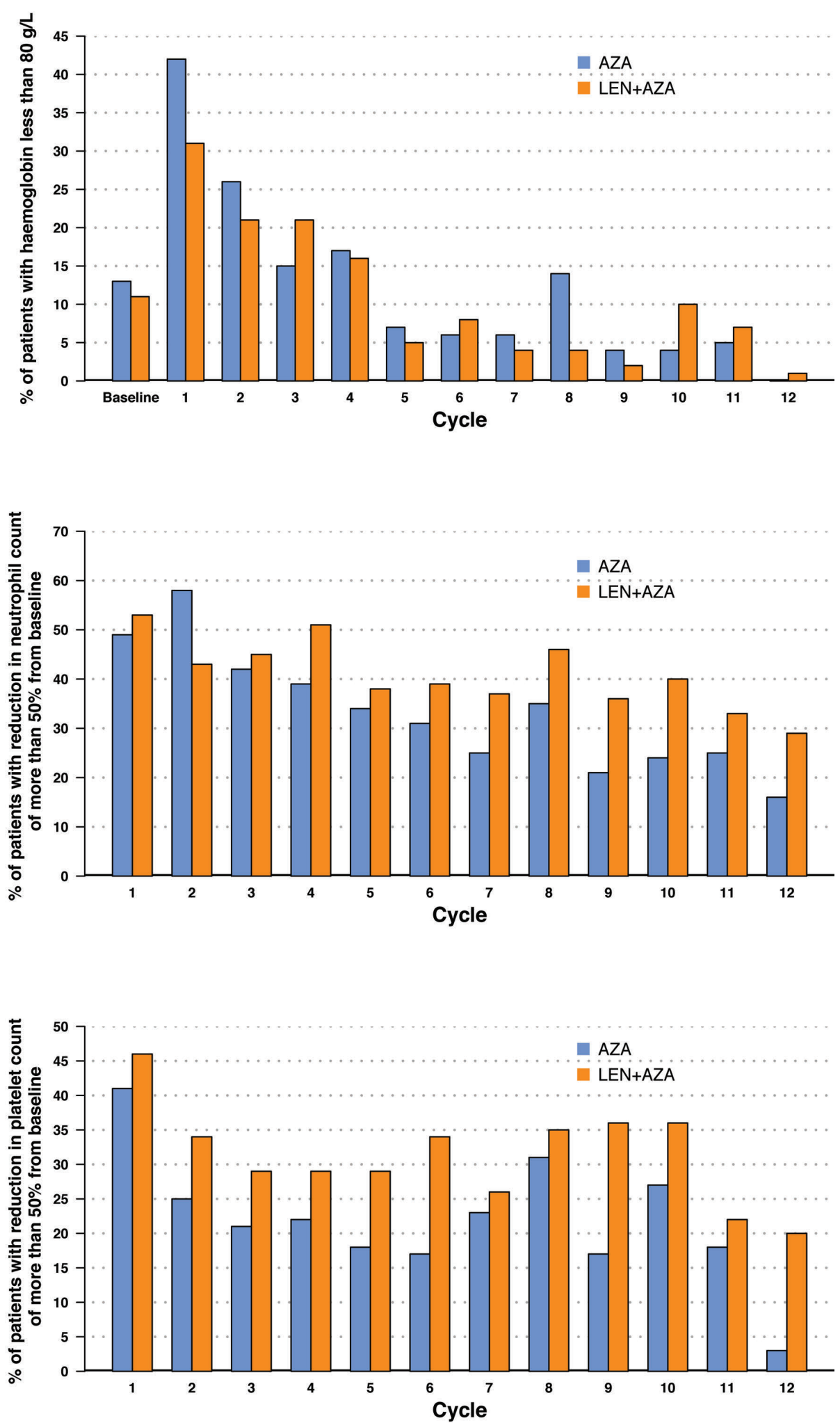

Figure 1. Rates of Grade 3+ Anemia ( $\mathrm{Hb}$ less than $80 \mathrm{~g} / \mathrm{L}$ ) baseline and on treatment, rates of Grade 3+ neutropenia (reduction neutrophils to less than $50 \%$ baseline) and rates of Grade $3+$ thrombocytopenia (reduction in platelets to less than $50 \%$ baseline). 
only $2.6 \%$ cycles dose- reduced. For those on combination treatment the median duration of lenalidomide treatment was 9 cycles (range 1-12) with only $2.8 \%$ lenalidomide cycles dose- reduced. Early discontinuation of azacitidine was mainly due to investigator/patient decision, relapse/progressive disease, or death. Early discontinuation of lenalidomide was mainly due to toxicity (11 patients) or investigator/patient decision (9 patients). Six patients were treated with lenalidomide after completing the protocol-specified 12 months of study therapy; 2 in AZA arm and 4 in LEN+AZA. This was initiated by individual investigators, and continued for up to 2 years post study therapy. The extended lenalidomide treatment was associated with grade 3 diarrhea in one patient and resulted in no improvement in response in these patients.

\section{Safety}

Non hematologic toxicity. Rates of all adverse events grade 3 or higher according to system and treatment arm are summarised in Online Supplementary Table S1 with no differences observed. The most common non-hematologic toxicity was infection; the overall number of infectious episodes grade 3 or worse was 132 in $42.8 \%$ patients.

There was no difference between the arms for overall rates of infection with sepsis being the most common infection type. The difference between the severity of sepsis between the two treatment arms was significant with greater severity in the combination arm; sepsis Grade 4+ was seen in 11 patients in the combination arm compared to 2 patients in azacitidine alone arm (Table 3). There were 17 deaths due to

Table 2. Molecular characteristics of cohort with baseline samples.

AZA (n=35) IEN-AZA (n=31) TOTAL (n=66)

Targeted Amplicon Sequencing

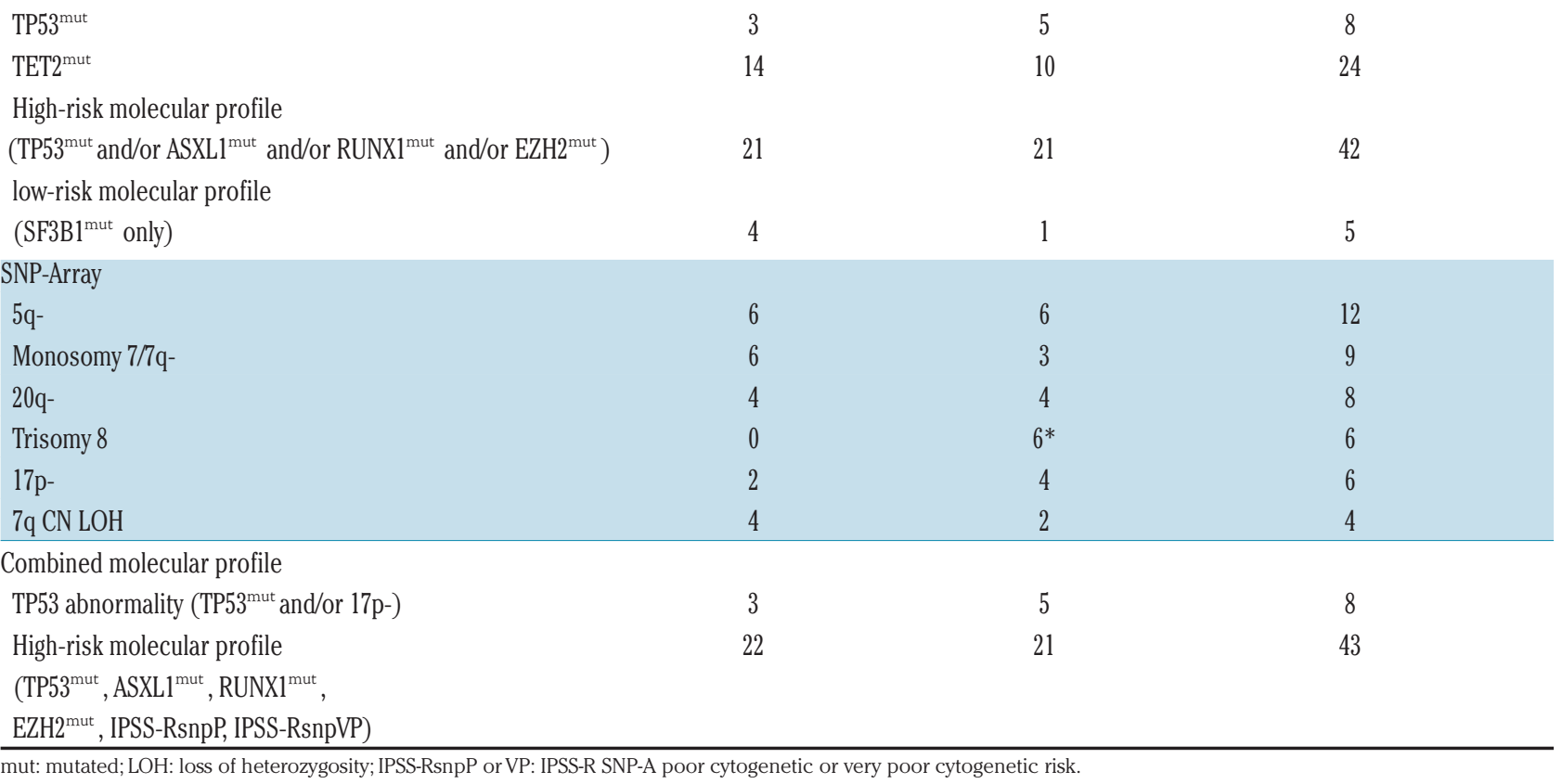

Table 3. Non-hematologic toxicity; infections, grade 3 and above.

\begin{tabular}{|c|c|c|c|c|}
\hline \multirow[t]{2}{*}{ Infection type } & \multicolumn{2}{|c|}{ AZA } & \multicolumn{2}{|c|}{ LEN+AZA } \\
\hline & N. patients & No. episodes & N patients & No. episodes \\
\hline Any Infection & $34(43 \%)$ & 62 & $34(43 \%)$ & 71 \\
\hline GI/Abdo & $6(8 \%)$ & 7 & $7(9 \%)$ & 10 \\
\hline Renal/Urologic & $0(0 \%)$ & 0 & $2(3 \%)$ & 2 \\
\hline Respiratory & $9(11 \%)$ & 10 & $14(18 \%)$ & 18 \\
\hline Sepsis overall & $18(23 \%)$ & 29 & $19(24 \%)$ & 29 \\
\hline Sepsis grade 3 & 16 & & 8 & \\
\hline Sepsis grade 4 & 2 & & 10 & $P=0.02$ \\
\hline Sepsis grade 5 & 0 & & 1 & \\
\hline Skin/Mucosal/Eye & $9(11 \%)$ & 11 & $9(11 \%)$ & 9 \\
\hline All other infections & $5(6 \%)$ & 5 & $6(8 \%)$ & 6 \\
\hline
\end{tabular}


infection (7 in AZA, 10 in LEN+AZA). For full listing of cause of death according to treatment arm see Online Supplementary Table S2.

The only other non hematologic toxicity seen at rates $>5 \%$ was raised GGT in 15 patients with no significant difference between the arms (AZA $n=4$ LEN+AZA $n=11 ; P=0.1$ ).

Hematologic toxicity. Comparing data from cycle 3 to cycle 12 (as LEN was introduced from cycle 3 onwards), there was no association between treatment arm and cycle for any of the hematologic grade $3+$ toxicity rates was observed. For $\mathrm{Hb}<80 \mathrm{~g} / \mathrm{L}$ and for neutrophils and platelets $>50 \%$ reduction from baseline count, there was no difference between treatment arms but a statistical significant difference across the cycles for both arms. See Figure 1.

Emerging Grade 3+ hematologic toxicity. For those patients who did not have a grade $3+$ toxicity at baseline as defined by CT CAE V4.0 (N), there was a non significant trend to greater treatment emergent neutropenia (78\% vs. 68\%) and thrombocytopenia ( $63 \%$ vs. $50 \%$ ) in the combination arm (Online Supplementary Table S3).

\section{Efficacy}

Summary of efficacy endpoints is provided in Table 4.

The primary endpoint of rate of clinical benefit at 12 months (alive with stable disease or better) in the AZA arm was $65 \%$, and $54 \%$ in the LEN+AZA arm (Fishers Exact test, $P=0.2)$. There was no difference in rate of clinical benefit between each treatment arm according to WHO diagnostic subgroup (MDS, AML or CMML) or according to IPSS-R.

There was no difference in clinical benefit across disease subtype within either the LEN+AZA or the AZA treated groups.

The overall response rate (best response of $\mathrm{HI}, \mathrm{PR}$, marrow CR or CR) with AZA was $57 \%$ and $69 \%$ in LEN+AZA $(P=0.14)$.

CR was achieved in 17 patients $(22 \%)$ on AZA and 20 patients $(25 \%)$ on LEN+AZA.

There was no difference in type of $\mathrm{HI}$ across the 2 arms.

The median time to best response for those achieving a HI or better was not different between treatment arms; 5.5 months (range 1.8-11.7) AZA and 4.8 months (range 1.8-12.4) LEN+AZA. Median time to first response (of HI or better) was 2.8 months (range 1.6-9.2), with no difference between the arms $(P=0.13)$.

There were no significant associations found for these variables with respect to response - either clinical benefit at 12 months as defined by primary endpoint, or for overall response rate of best response HI or better.

Using univariable subgroup logistic regression models of treatment effect on primary endpoint response (clinical benefit stable disease or better at 12 months), there were no significant associations for age, sex, WHO diagnosis, IPSS, IPSS-R or cytogenetic risk group (Online Supplementary Figure S1).

\section{Cytogenetic response}

Fifty-nine patients had a karyotypic abnormality detected at baseline, 28 in the AZA arm and 31 in the LEN+AZA arm. A total of $29 \%$ (8) of patients on the AZA arm had a cytogenetic response. Half (4) of those achieved a complete cytogenetic response while the other half (4) achieved a partial response with a $\geq 50 \%$ reduction in the chromosomal abnormality. A total of $39 \%$ (12) patients in the LEN+AZA arm had a cytogenetic response, 11 of them
A
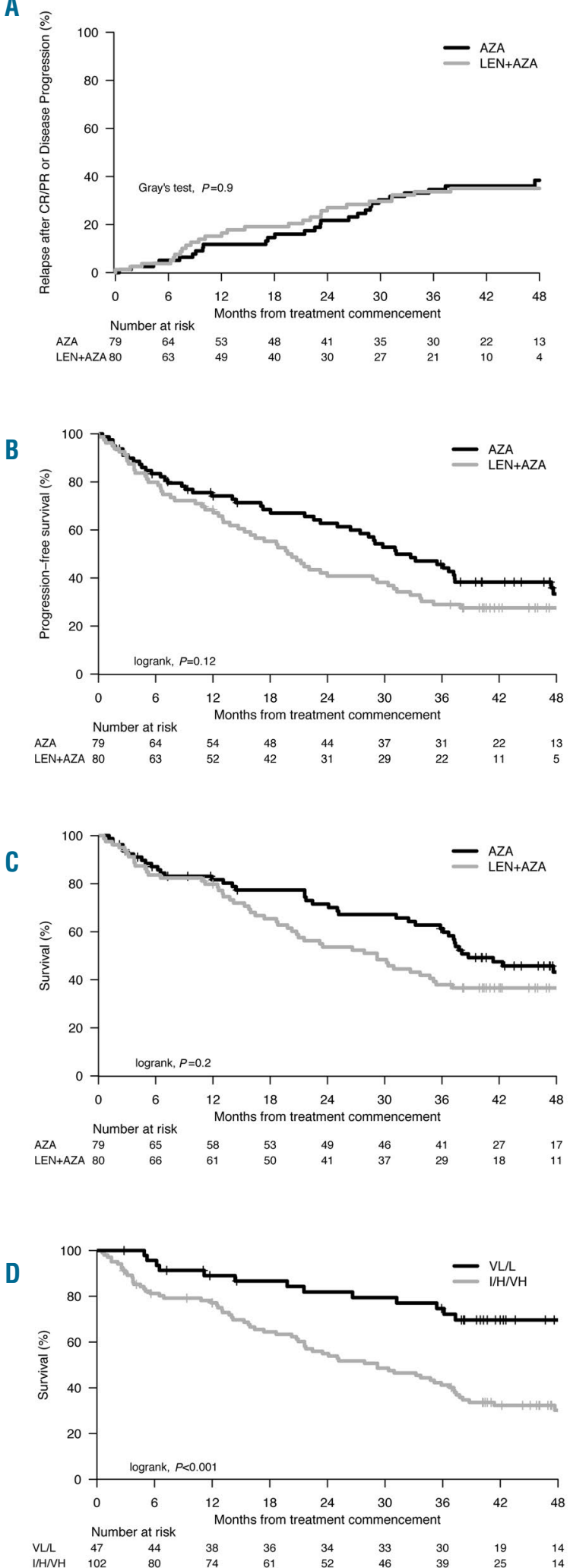

Figure 2. Time to relapse, progression-free and overall survival between treatment cohorts, and overall survival according to risk. A. Kaplan-Meier curves of time to relapse after achieving CR/PR, or disease progression between both treatment cohorts. B. Kaplan-Meier curves of progression-free survival (PFS). C. Overall survival according to assigned treatment cohort. D. Overall survival according to IPSS-R; very low/low versus intermed/high/very high risk. 
achieving a complete cytogenetic response whilst 1 achieved a partial response..

Time to disease relapse or progression (Figure 2A)

No association with treatment arm was found for time to relapse after CR/PR or PD.

Median time to progression to AML (MDS and CMML patients by WHO criteria) or death (all patients) from any cause was 37.2 months in the AZA arm and 28.8 months in the LEN+AZA arm.

\section{Progression free-survival (PFS) (Figure 2B)}

PFS was measured from first day of treatment to date of first confirmed disease progression or death from any cause. Median PFS time on AZA was 31.2 months $(95 \%$ CI 25.0-37.4) and on LEN+AZA was 19.8 months (95\% CI 14.7-29.2) with no difference between the arms observed (Figure 2B, logrank $P=0.12$ ).

\section{Overall survival (Figures 2C and 2D)}

The median follow-up time (estimated with the inverse Kaplan-Meier method) was 47.2 months (range 0.7-59.5); median survival time on AZA was 38.8 months $(95 \% \mathrm{CI}$ 35.8-52.6) compared to 29.2 months on LEN+AZA (95\%CI 19.8-35.1) (logrank $P=0.2$ ). Forty-one patients on AZA had died compared to 49 on LEN+AZA (Table 8). Cause of death was mostly due to disease progression and infections with 5 overall due to hemorrhage and 9 other/unknown. There was a significant difference in median overall survival in IPSS-R Very Low/Low-risk and Intermediate/High/Very High-risk groups (Figure 2D, logrank $P<0.001$ ).

\section{Quality of Life (Figure 3)}

Completion rates for the EORTC OLQ-C30 at baseline/screening was 96\%, at C4D22 83\%, C8D22 82\% and C12D22 or at primary endpoint visit was $84 \%$. The only effect of treatment on QoL scores during study was a higher rate of diarrhea in LEN+AZA arm.

\section{Discussion}

This randomized phase II study aimed to find out whether outcomes were improved for patients with higher risk MDS, CMML and low blast AML by adding lenalidomide treatment to the established regimen of azacitidine. These two agents have shown synergistic activity in vitro, with promising early results of the combination treatment from smaller single arm clinical trials. In this study, there was excellent duration and delivery of treatment in both arms due to strong recognition of the value of prolonged therapy, particularly with a clinical benefit endpoint at 12 months. Despite this and the good tolerability of the combination, there was no improvement in response rates, clinical benefit or survival. As in the study by Sekeres et al., ${ }^{21}$ we showed a trend towards improved responses without translation to improved clinical benefit or survival, though this study was not adequately powered to show a difference in overall survival. The lack of clinical benefit was not due to an excess of toxicity in the combination arm.

No subgroup in this study, including those with CMML, and in contrast to the recent report by Sekeres et al. ${ }^{21}$ had improved responses with the addition of lenalidomide to

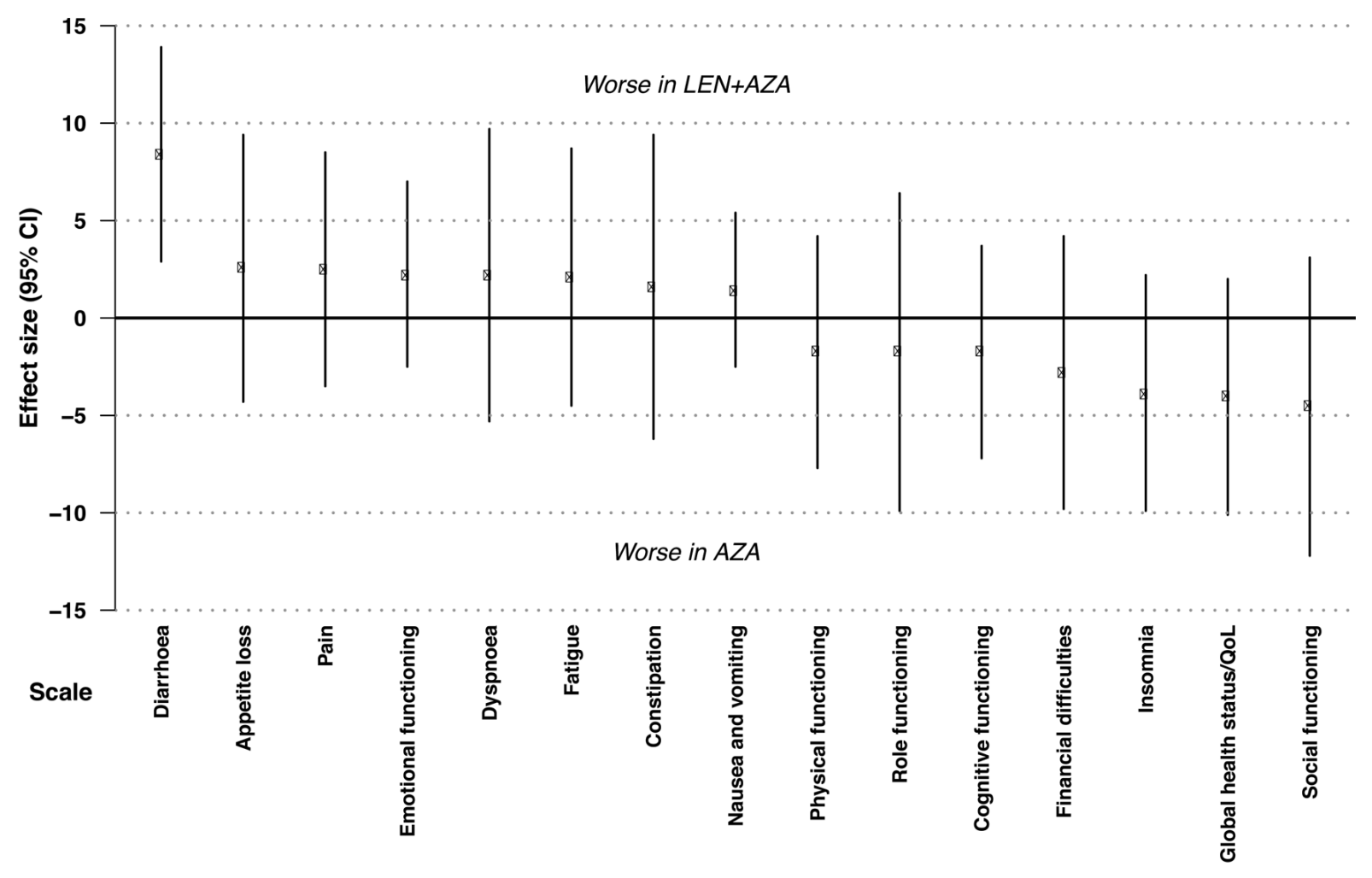

Figure 3. Quality of life differences on EORTC QLQ C30 questionnaire between treatment cohorts using GEE regression model 
Table 4. Efficacy: clinical benefit at 12 months, overall response rate (ORR) \& best response achieved by assigned treatment cohort; those who received treatment.

\begin{tabular}{|c|c|c|c|}
\hline & $\begin{array}{c}\text { AZA n }=79 \\
\text { n (\%[Exact } 95 \% \text { Cl] })\end{array}$ & $\begin{array}{c}\text { LEN+AZA } n=80 \\
n(\%[\text { Exact } 95 \% \text { CI]) }\end{array}$ & $\boldsymbol{P}$ \\
\hline Clinical benefit at 12 months (SD or better) & $52(65 \%[54-75])$ & $43(54 \%[42-65])$ & 0.2 \\
\hline MDS & $38(63 \%$ [50-75]) & $34(58 \%[44-70])$ & 0.6 \\
\hline AML & $5(62 \%[24-91])$ & $4(36 \%[11-69])$ & 0.4 \\
\hline \multirow[t]{2}{*}{ CMML } & $9(75 \%[43-95])$ & $5(50 \%[19-81])$ & 0.4 \\
\hline & $P=0.7$ & $P=0.4$ & \\
\hline IPSS-R Very Low/Low & $22(85 \%[65-96])$ & $12(57 \%[34-78])$ & 0.052 \\
\hline IPSS-R Intermed/High/Nery High & $28(57 \%[42-71])$ & $28(52 \%[38-66])$ & 0.7 \\
\hline Overall response rate (Best response) & $45(57 \%[45-68])$ & $55(69 \%$ [57-79]) & 0.14 \\
\hline MDS & $36(60 \%[47-72])$ & $41(69 \%[56-81])$ & 0.3 \\
\hline AML & $3(43 \%[10-82])$ & $6(55 \%[23-83])$ & $>0.99$ \\
\hline CMML & $6(50 \%[21-79])$ & $8(80 \%[44-97])$ & 0.2 \\
\hline IPSS-R Very Low/Low & $15(58 \%[37-77])$ & $14(67 \%$ [43-85]) & 0.6 \\
\hline IPSS-R Intermed/High/Nery High & $28(58 \%[43-72])$ & $37(69 \%[54-80])$ & 0.3 \\
\hline \multicolumn{4}{|l|}{ Best response achieved } \\
\hline $\mathrm{CR}$ & $17(22 \%)$ & $20(25 \%)$ & \\
\hline PR & 0 & $2(2 \%)$ & \\
\hline Marrow CR & $2(2 \%)$ & $5(6 \%)$ & \\
\hline Marrow $\mathrm{CR}+\mathrm{HI}$ & $8(10 \%)$ & $5(6 \%)$ & \\
\hline HI only & $18(23 \%)$ & $23(29 \%)$ & \\
\hline SD & $22(28 \%)$ & $15(19 \%)$ & \\
\hline PD & $3(4 \%)$ & $4(5 \%)$ & \\
\hline Death prior to C3 first response assessment & $6(8 \%)$ & $4(5 \%)$ & \\
\hline Missing data/not evaluable & $3(4 \%)$ & $2(2 \%)$ & \\
\hline
\end{tabular}

CR: complete response; HI: hematologic improvement; PD: progressive disease; PR: partial response; SD: stable disease.

azacitidine. Overall, there was very good durability of responses and good survival. Unsurprisingly, those with lower-risk disease according to established prognostic scores lived longer.

Our eligibility included patients with potentially lowerrisk disease subtypes in contrast to other recent clinical trials such as AZAO01 ${ }^{9}$ and SWOG S1117 ${ }^{21}$ which defined eligibility based on prognostic score. This inclusion was based on earlier data showing similar response rates across all IPSS groups ${ }^{7}$ and an acknowledgement that a proportion of those with apparent lower-risk disease have outcomes more in keeping with those with higher prognostic scores. Despite this, and though it is an indirect comparison of populations, our cohort risk compares similarly to the SWOG S1117 cohort with respect to proportion of patients with Very Low/Low IPSS-R; 31.3\% Sekeres cohort compared to our ALLG MDS4 32\% (AZA) and $38 \%$ (AZA + LEN).

It is possible that the dose and scheduling of treatment in this protocol may have impacted on responses. Phase 1 data by Sekeres ${ }^{26}$ supported the decision to reduce the number of days of azacitidine dosing to five when combining with lenalidomide, in order to reduce the risk of treatment limiting toxicity in the first two cycles. Given the lack of excessive toxicity in our combination arm and the high median number of azacitidine cycles (11 cycles in our cohort compared to SWOG 1117 median 23-25 weeks treatment) we did achieve the implementation of this treatment combination on a broad multi-centre setting. However, we have not shown that full azacitidine dosing of seven days per cycle in combination with lenalidomide is feasible. In addition, the concurrent as opposed to consecutive administration of the two agents on this protocol may have reduced overall efficacy. The dose of lenalidomide selected for this study was based on a Phase II study in $\mathrm{MDS},{ }^{21}$ however, subsequent studies have utilised higher doses of lenalidomide in combination - mostly sequential - in $\mathrm{AML}^{30}$ and high-risk $\mathrm{MDS}^{31}$ which may improve efficacy. The option of using lenalidomide prior to the introduction of azacitidine could be considered as an extrapolation of the findings by Zeidan et al. who demonstrated enhanced erythroid improvement in low -risk (non-5q deletion) MDS. ${ }^{31}$ Finally, consideration could be given to commencing both agents from $\mathrm{C} 1$ rather than delaying the introduction of lenalidomide until C3 in an attempt to improve efficacy, although early progressions or deaths in our study were uncommon with 10 deaths or disease progression within the first 2 cycles of treatment (5 in each arm).

There was no central review of pathology in this study. Responses were provided by the site investigators and only reviewed centrally if there were discrepancies or questions. IWG criteria for response was adopted, though its application in patients experiencing both disease and treatment related cytopenias is complex, and the application and consistency across many sites was 
difficult to ensure. A more robust, refined IWG criteria is awaited and would make this more consistent in future studies.

We have shown the feasibility on a broad scale of the combination of lenalidomide and azacitidine in patients with higher risk MDS, CMML and low blast count AML, but the lack of improvement in responses and clinical benefit do not support the utilization of this combination in higher-risk MDS in clinical practice. Other combinations and novel agents are needed to improve the outcomes for this large and vulnerable group of patients, who at this stage have limited therapeutic options. Other groups are currently exploring novel combination studies with azacitidine such as enasidinib and venetoclax.

\section{Acknowledgments}

The authors wish to acknowledge the Australasian Leukaemia \& Lymphoma Group for conduct of the study, all site investigators and study personnel. We also wish to thank our patients and families.

\section{Funding}

This study was supported by Celgene with grant funding for study conduct, Snowdome Foundation and the Victorian Epigenetic Group for funding support for molecular studies.

\section{References}

1. Arber DA, Orazi A, Hasserjian R, et al. The 2016 revision to the World Health Organization Classification of Myeloid neoplasms and Acute leukemia. Blood. 2016;127(20):2391-2405

2. Gangat N, Patnaik MM, \& Tefferi A. Myelodysplastic syndromes: Contemporary Review and how we treat. Am J Hematol. 2016;91(1):76-89.

3. Shukron O, Vainstein V, Kündgen A, Germing U, \& Agur Z. Analyzing transformation of myelodysplastic syndrome to secondary acute myeloid leukemia using a large patient database. Am J Hematol. 2012;87(9):853-860.

4. Greenberg PL, Tuechler H, Schanz J, et al. Revised International Prognostic Scoring System for myelodysplastic syndromes. Blood. 2012;120(12):2454-2465.

5. Greenberg P, Cox C, LeBeau, et al. International Scoring System for evaluating prognosis in myelodysplastic syndromes. Blood. 1997;89(6):2079-2088.

6. Christman JK. 5-Azacytidine and 5-aza-2'deoxycytidine as inhibitors of DNA methylation: mechanistic studies and their implications for cancer therapy. Oncogene. 2012;21(35):5483-5495.

7. Silverman LR, Demakos E. Peterson B, et al. Randomized controlled trial of azacitidine in patients with the myelodysplastic yndrome: a study of the Cancer and Leukemia Group B. J Clin Oncol. 2002;20(10):2429-2440.

8. Kornblith AB, Herndon JE, Silverman LR, et al. Impact of azacytidine on the quality of life of patients with myelodysplastic syndrome treated in a randomized phase III trial: a cancer and leukemia group B study. J Clin Oncol. 2002;20(10):2441-2452.

9. Fenaux P, Mufti GJ, Hellstrom E, et al. Efficacy of azacitidine compared with that of conventional care regimens in the treatment of higher-risk myelodysplastic syndromes: a randomised, open-label, phase III study. Lancet Oncol. 2009;10(3):223-232.

10. Dombret H, Seymour JF, Butrym A, et al. International phase 3 study of Azacitidine vs conventional care regimens in older patients with newly diagnosed AML with $>30 \%$ blasts. Blood. 2015;126(3):291-299.

11. Fenaux P, Mufti,GJ, Hellstrom E, et al. Azacitidine prolongs overall survival compared with conventional care regimens in elderly patients with low bone marrow blast count acute myeloid leukemia. J Clin Oncol. 2010;28(4):562-569.

12. Ball B, Zeidan A, Gore SD \& Prebet T. Hypomethylating agent combination strategies in myelodysplastic syndromes: hopes and shortcomings. Leuk Lymphoma. 2017:58(5):1022-1036.

13. Rami SK, Sallman D, Ali M, et al. Outcome of myelodysplastic syndrome patients with TP53 mutation treated with hypomethylating agents. Blood. 2017;130(Suppl 1):1687.

14. Kotla V, Goel S, Nischal S, et al. Mechanism of action of lenalidomide in hematological malignancies. J Hematol Oncol. 2009;2:36

15. List A, Kurtin S, Roe DJ, et al. Efficacy of lenalidomide in myelodysplastic syndromes. N Engl J Med. 2005;352(6):549557.

16. Fenaux P, Giagounidis A, Selleslag D, et al. A randomized phase 3 study of lenalidomide versus placebo in RBC transfusion dependent patients with low-/intermediate-1-risk myelodysplastic syndromes with del5q. Blood. 2011; 118(14):3765-3776

17. Raza A, Reeves JA, Feldman EJ, et al. Phase 2 study of lenalidomide in transfusiondependent, low-risk, and intermediate-1 risk myelodysplastic syndromes with karyotypes other than deletion 5q. Blood 2008;111(1):86-93

18. Santini V, Almeida A, Giagounidis A, et al Randomized phase III study of lenalidomide versus placebo in RBC transfusiondependent patients with lower-risk non$\operatorname{del}(5 q)$ myelodysplastic syndromes and ineligible for or refractory to erythropoiesis-stimulating agents. J Clin Oncol. 2016;34(25):2988-2996.

19. Sibon D, Cannas G, Baracco F. et al. Lenalidomide in lower-risk myelodysplastic syndromes with karyotypes other than deletion $5 q$ and refractory to erythropoiesis-stimulating agents. Br J Haematol. 2012;156(5):619-625.

20. Kenealy M, Patton N, Filshie $R$, et al. Results of a phase II study of thalidomide and azacitidine in patients with clinically advanced myelodysplastic syndromes (MDS), chronic myelomonocytic leukemia (CMML) and low blast count acute myeloid leukemia (AML). Leuk Lymphoma. 2017;58(2):298-307.

21. Sekeres MA, Othus M, List AF, et al. Randomized phase II study of azacitidine alone or in combination with lenalidomide or with vorinostat in higher-risk myelodysplastic syndromes and chronic myelomonocytic leukemia: North American Intergroup Study SWOG S1117. J Clin Oncol. 2017;35(24):2745-2753.

22. Narayan R, Garcia JS, Percival MM, et al Sequential azacitidine plus lenalidomide in previously treated elderly patients with acute myeloid leukemia and higher risk myelodysplastic syndrome. Leuk Lymphoma. 2016;57(3):609-615.

23. DiNardo CD, Daver N, Jabbour et al. Sequential azacitidine and lenalidomide in patients with high-risk myelodysplastic syndromes and acute myeloid leukemia: a single arm, phase $1 / 2$ study. Lancet Haematol. 2015;2(1):e12-e20.

24. Swerdlow SH, Campo E, Harris NL, et al. WHO Classification of Tumours of Haematopoietic and Lymphoid Tissues 4th Edition. International Agency for Research on Cancer (2008)

25. Lyons RM, Cosgriff TM, Modi SS, et al. Hematologic response to three alternative dosing schedules of azacitidine in patients with myelodysplastic syndromes. J Clin Oncol. 2009;27(11):1850-1856.

26. Sekeres MA, List AF, Cuthbertson D, et al Phase I combination trial of lenalidomide and azacitidine in patients with higher-risk myelodysplastic syndromes. J Clin Oncol. 2010;28(13):2253-2258

27. Cheson BD, Greenberg PL, Bennett J, et al. Clinical application and proposal for modification of the International Working Group (IWG) response criteria in myelodysplasia. Blood. 2006;108(2):419425

28. Bejar R. Clinical and genetic predictors of prognosis in myelodysplastic syndromes. Haematologica. 2014;99(6):956-964.

29. Kulasekararaj AG, Mohamedali AM, \& Mufti GJ. Recent advances in understanding the molecular pathogenesis of myelodysplastic syndromes. Br J Haematol. 2013;162(5):587-605

30. Medeiros BC, McCaul K, Kambhampati S et al. Randomized study of continuous high dose lenalidomide, sequential azacitidine and lenalidomide, or azacitidine in persons 65 years and over with newly diagnosed acute myeloid leukemia. Haematologica. 2018;103(1):101-106.

31. Zeidan AM, Al Ali NH, Padron E, et al. Lenalidomide treatment for lower risk nondeletion $5 \mathrm{q}$ myelodysplastic syndromes patients yields higher response rates when used before Azacitidine. Clin Lymphoma Myeloma Leuk. 2015;15(11):705-710 


\section{University Library}

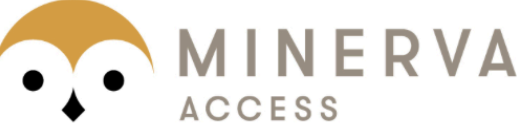

A gateway to Melbourne's research publications

Minerva Access is the Institutional Repository of The University of Melbourne

\section{Author/s:}

Kenealy, M;Hertzberg, M;Benson, W;Taylor, K;Cunningham, I;Stevenson, W;Hiwase, D;Eek, R;Zantomio, D;Jong, S;Wall, M;Blombery, P;Gerber, T;Debrincat, M;Zannino, D;Seymour, JF

Title:

Azacitidine with or without lenalidomide in higher risk myelodysplastic syndrome \& low blast acute myeloid leukemia

Date:

2019-03-31

\section{Citation:}

Kenealy, M., Hertzberg, M., Benson, W., Taylor, K., Cunningham, I., Stevenson, W., Hiwase, D., Eek, R., Zantomio, D., Jong, S., Wall, M., Blombery, P., Gerber, T., Debrincat, M., Zannino, D. \& Seymour, J. F. (2019). Azacitidine with or without lenalidomide in higher risk myelodysplastic syndrome \& low blast acute myeloid leukemia. HAEMATOLOGICA, 104 (4), pp.700-709. https://doi.org/10.3324/haematol.2018.201152.

Persistent Link:

http://hdl.handle.net/11343/253382

License:

CC BY-NC 